\title{
Is Cross-reactivity with Nontuberculous Mycobacteria a Systematic Problem in the Xpert MTB/RIF Assay?
}

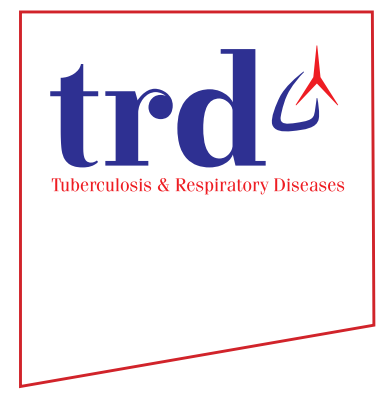

\author{
Hee Jae Huh, M.D. ${ }^{1}{ }^{\mathbb{D}}$, Dong Joon Song ${ }^{1}$, Chang-Seok Ki, M.D. ${ }^{2}$ and Nam Yong Lee, M.D. ${ }^{1}$ (iD \\ ${ }^{1}$ Department of Laboratory Medicine and Genetics, Samsung Medical Center, Sungkyunkwan University School of Medicine, \\ Seoul, ${ }^{2}$ Green Cross Genome, Yongin, Korea
}

The Xpert MTB/RIF assay (Xpert assay; Cepheid, Sunnyvale, CA, USA) is a fully automated, real-time polymerase chain reaction assay that is designed to detect the presence of Mycobacterium tuberculosis (MTB) and rifampin (RFP) resistance ${ }^{1}$. The Xpert assay can be utilized as a rapid diagnostic test for tuberculosis and RFP-resistant tuberculosis ${ }^{1}$. The review article which was recently published in this journal emphasized rapid molecular diagnosis of RFP-resistant tuberculosis for the optimal treatment of multidrug-resistant tuberculsosis ${ }^{2}$.

Xpert assay can also be used to differentiate MTB from nontuberculous mycobacteria (NTM) in acid-fast bacilli (AFB) smear-positive cases ${ }^{3}$. Although several previous studies have demonstrated that the Xpert assay showed excellent performance in distinguishing MTB from NTM in smear-positive or negative clinical specimens ${ }^{1,4}$, the misdiagnosis of MTB by the Xpert assay was recently reported for five NTM species (M. abscessus, M. marinum, M. smegmatis, M. phlei, and M. aurum) at a high bacterial load ${ }^{5}$. The results reported by Pang et al. ${ }^{5}$ have very important clinical implications, because AFB smear microscopy cannot differentiate between MTB and NTM and many patients with NTM pulmonary disease were misdiagnosed with pulmonary tuberculosis and even mul-

Address for correspondence: Nam Yong Lee, M.D.

Department of Laboratory Medicine and Genetics, Samsung Medical Center, Sungkyunkwan University School of Medicine, 81 Irwon-ro, Gangnam-gu, Seoul 06351, Korea

Phone: 82-2-3410-2706, Fax: 82-2-3410-2719

E-mail: micro.lee@samsung.com

Received: Sep. 15, 2018

Revised: Oct. 4, 2018

Accepted: Oct. 23, 2018

(c) It is identical to the Creative Commons Attribution Non-Commercial License (http://creativecommons.org/licenses/by-nc/4.0/). The Korean Academy of Tuberculosis and Respiratory Diseases. tidrug-resistant tuberculosis ${ }^{6}$. Moreover, $M$. abscessus is an important etiologic organism of NTM pulmonary disease in many countries with high tuberculosis burden such as China and South Korea ${ }^{7,8}$. Misdiagnosis of NTM as MTB or RFPresistant MTB is a significant concern in defining a proper tuberculosis program in these countries. We therefore tried to validate the previous experiment with the misdiagnosed NTM species to evaluate whether the Xpert assay showed crossreactivity between MTB and five NTM species.

A total of five NTM reference strains (M. abscessus, M. marinum, M. smegmatis, M. phlei, and M. aurum) from American Type Culture Collection or the Korean Mycobacteria Resource Center were included in this study (Table 1). Freshly grown bacterial colonies were obtained from 3\% Ogawa agar (Shinyang, Seoul, Korea). Subsequently, the bacteria were suspended in sterile distilled water and vortexed vigorously. The turbidity was adjusted to a $0.5 \mathrm{McF}$ arland (approximately $1 \times 10^{7}$ colony-forming unit $\left.[\mathrm{CFU}] / \mathrm{mL}\right)^{9}$. Additionally, the bacteria were diluted, resulting in tubes containing $1 \times 10^{5} \mathrm{CFU} /$ $\mathrm{mL}$ equivalents. The Xpert assay was performed using the G4 version of cartridges with sediment protocol according to the

Table 1. Xpert assay results for detecting different mycobacterial species

\begin{tabular}{|llcc|}
\hline \multicolumn{1}{c}{ Species } & \multicolumn{1}{c}{$\begin{array}{c}\text { Strain(s) and } \\
\text { source }\end{array}$} & $\begin{array}{c}\text { Xpert result at different } \\
\text { bacterial loads } \\
\text { (CFU/mL equivalent) }\end{array}$ \\
\cline { 3 - 4 } M. abscessus & ATCC19977 & ND & ND \\
M. aurum & ATCC51345 & ND & ND \\
M. marinum & KMRC 00136-21108 & ND & ND \\
M. phlei & ATCC11758 & ND & ND \\
M. smegmatis & ATCC19420 & ND & ND \\
\hline
\end{tabular}

CFU: colony-forming unit; ATCC: American Type Culture Collection; ND: not detected; KMRC: Korean Mycobacteria Resource Center. 
manufacturer's instructions.

The Xpert results for different NTM species at two concentrations are summarized in Table 1. All NTM species gave negative results, and non-specific positive reactions were not observed in any probes: no changes in threshold cycle value were observed. The sample processing controls in all tested bacterial suspensions met the acceptance criteria, and no invalid results were observed for any sample.

The manufacturer presented the data for analytical specificity and exclusivity in the package inser ${ }^{10}$. A total of 24 NTM species, including $M$. abscessus, M. marinum, and M. smegmatis were tested at $10^{8} \mathrm{CFU} / \mathrm{mL}$ or $10^{6} \mathrm{CFU} / \mathrm{mL}$. No MTB results were generated from NTM strains except $M$. scrofulaceum at $10^{8} \mathrm{CFU} / \mathrm{mL}$ (one of three replicates). Moreover, numerous studies provided data on a variety of NTM that grew from the specimens tested to look for evidence of crossreactivity ${ }^{1}$. A systematic review on the diagnostic accuracy of the Xpert assay reported that only one specimen $(0.6 \%)$ exhibited a false-positive result among 180 specimens that grew $\mathrm{NTM}^{1}$. On the other hand, Pang et al. ${ }^{5}$ reported that misdiagnosis of MTB, even RFP-resistant MTB by the Xpert assay was observed in five NTM species at a high bacterial load, although these results were obtained on the basis of artificial non-human samples. They regarded this cross-reactivity as an intrinsic problem of the Xpert assay. A high burden of organisms might cause fluorescence cross-talk in the detection channels, which could lead to false-positive results. However, other possible causes of false-positivity should be excluded such as specimen contamination at the time of sample preparation, since an artificial bacterial suspension was used. Indeed, we could not reproduce their findings in our study: we did not observe any false-positive reactions in the five NTM species, even at a higher concentration of $1 \times 10^{7} \mathrm{CFU} / \mathrm{mL}$ equivalents. In light of these data, it is difficult to interpret the false-positive results for five NTM species at a high bacterial load as a systematic cross-reactivity issue in the Xpert assay. However, our study has limitations. Since only one strain per species was used in this study, we cannot exclude the possibility of cross-reactivity with some NTM strains.

In conclusion, we tested the Xpert assay for cross-reactivity between MTB and five NTM species at high bacterial load and did not observe any false-positive reactions. Therefore, the previously reported cross-reactivity between MTB and five NTM species may not be a systematic problem with the Xpert assay.

\section{Authors' Contributions}

Conceptualization: Huh HJ, Ki CS, Lee NY. Writing - original draft preparation: Huh HJ. Writing - review and editing: all authors. Approval of final manuscript: all authors.

\section{Conflicts of Interest}

No potential conflict of interest relevant to this article was reported.

\section{References}

1. Steingart KR, Schiller I, Horne DJ, Pai M, Boehme CC, Dendukuri N. Xpert(R) MTB/RIF assay for pulmonary tuberculosis and rifampicin resistance in adults. Cochrane Database Syst Rev 2014;(1):CD009593.

2. Jeon D. WHO treatment guidelines for drug-resistant tuberculosis, 2016 update: applicability in South Korea. Tuberc Respir Dis 2017;80:336-43.

3. Huh HJ, Jeong BH, Jeon K, Koh WJ, Ki CS, Lee NY. Performance evaluation of the Xpert MTB/RIF assay according to its clinical application. BMC Infect Dis 2014;14:589.

4. Marlowe EM, Novak-Weekley SM, Cumpio J, Sharp SE, Momeny MA, Babst A, et al. Evaluation of the Cepheid Xpert MTB/RIF assay for direct detection of Mycobacterium tuberculosis complex in respiratory specimens. J Clin Microbiol 2011;49:1621-3.

5. Pang Y, Lu J, Su B, Zheng H, Zhao Y. Misdiagnosis of tuberculosis associated with some species of nontuberculous mycobacteria by GeneXpert MTB/RIF assay. Infection 2017;45:677-81.

6. Ryu YJ, Koh WJ, Daley CL. Diagnosis and treatment of nontuberculous mycobacterial lung disease: clinicians' perspectives. Tuberc Respir Dis 2016;79:74-84.

7. Pang Y, Tan Y, Chen J, Li Y, Zheng H, Song Y, et al. Diversity of nontuberculous mycobacteria in eastern and southern China: a cross-sectional study. Eur Respir J 2017;49:1601429.

8. Koh WJ, Jeong BH, Kim SY, Jeon K, Park KU, Jhun BW, et al. Mycobacterial characteristics and treatment outcomes in Mycobacterium abscessus lung disease. Clin Infect Dis 2017;64:309-16.

9. Greendyke R, Byrd TF. Differential antibiotic susceptibility of Mycobacterium abscessus variants in biofilms and macrophages compared to that of planktonic bacteria. Antimicrob Agents Chemother 2008;52:2019-26.

10. Cepheid. Xpert MTB/RIF assay, 301-1404 Rev. B [package insert]. Sunnyvale: Cepheid; 2015. 